\title{
CT and MRI of the liver for hepatocellular carcinoma
}

\author{
Cynthia Santillan \\ Department of Radiology, University of California San Diego, San Diego, CA 92103, USA. \\ Correspondence to: Dr. Cynthia Santillan, Department of Radiology, University of California San Diego, 200 W Arbor Dr. MC \\ 8756, San Diego, CA 92103, USA. E-mail:csantillan@health.ucsd.edu \\ How to cite this article: Santillan C. CT and MRI of the liver for hepatocellular carcinoma. Hepatoma Res 2020;6:63. \\ http://dx.doi.org/10.20517/2394-5079.2020.60 \\ Received: 18 Jun 2020 First Decision: 23 Jul 2020 Revised: 30 Jul 2020 Accepted: 10 Aug 2020 Published: 1 Sep 2020 \\ Academic Editor: Yuko Kono Copy Editor: Cai-Hong Wang Production Editor: Jing Yu
}

\begin{abstract}
Computed tomography (CT) and magnetic resonance imaging (MRI) are commonly used modalities for the imaging based diagnosis and staging of hepatocellular carcinoma (HCC). The Liver Imaging Reporting and Data System (LI-RADS) was initially released in 2011 in an effort to standardize the interpretation and reporting of these studies in patients at increased risk for the development of HCC. With the release of LI-RADS V2018, LI-RADS has reached two important milestones - 10 years since the formation of the American College of Radiology supported LI-RADS committee and integration of LI-RADS into the 2018 American Association for the Study of Liver Disease practice guidance for HCC. In this article, we will discuss recent changes to LI-RADS with v2018, technical recommendations for the performance of $\mathrm{CT}$ and $\mathrm{MRI}$ in patients at risk for HCC, and critical imaging features in the LI-RADS algorithm.
\end{abstract}

Keywords: Hepatocellular carcinoma, Liver Imaging Reporting and Data System, magnetic resonance imaging, computed tomography

\section{INTRODUCTION}

LI-RADS was originally developed to provide a consistent method of conveying relative concern for the presence of hepatocellular carcinoma (HCC) or other malignancies based on Computed tomography(CT) or magnetic resonance imaging (MRI) of a patient at increased risk of developing $\mathrm{HCC}^{[1,2]}$. This relative concern for HCC, malignancy, or tumor in vein is conveyed by the different categories shown in Table 1 . Liver Imaging Reporting and Data System (LI-RADS) v2018 has now evolved to also include guidance 
Table 1. LI-RADS v2018 diagnostic categories

\begin{tabular}{ll}
\hline Category & \multicolumn{1}{c}{ Definition } \\
\hline LR-1 & Definitely benign \\
LR-2 & Probably benign \\
LR-3 & Intermediate probability of malignancy \\
LR-4 & Probably HCC \\
LR-5 & Definitely HCC \\
LR-M & Probably or definitely malignancy but not HCC specific \\
LR-TIV & Definite tumor in vein \\
LR-NC & Cannot be categorized due to image degradation or omission \\
\hline
\end{tabular}

LI-RADS: Liver Imaging Reporting and Data System; HCC: hepatocellular carcinoma; LR-TIV: LI-RADS Tumor in Vein

\section{CT/MRI Diagnostic Table}

\begin{tabular}{|c|c|c|c|c|c|c|}
\hline \multirow{2}{*}{\multicolumn{2}{|c|}{$\begin{array}{l}\text { Arterial phase hyperenhancement (APHE) } \\
\text { Observation size (mm) }\end{array}$}} & \multicolumn{2}{|c|}{ No APHE } & \multicolumn{3}{|c|}{ Nonrim APHE } \\
\hline & & $<20$ & $\geq 20$ & $<10$ & $10-19$ & $\geq 20$ \\
\hline \multirow{3}{*}{$\begin{array}{l}\text { Count additional major features: } \\
\text { - Enhancing "capsule" } \\
\text { - Nonperipheral "washout" } \\
\text { - Threshold growth }\end{array}$} & None & LR-3 & LR-3 & LR-3 & LR-3 & LR-4 \\
\hline & One & LR-3 & LR-4 & LR-4 & & LR-5 \\
\hline & $\geq$ Two & LR-4 & LR-4 & LR-4 & LR-5 & LR-5 \\
\hline
\end{tabular}

\section{LR-4 Observations in this cell are categorized based on one additional major feature: \\ - LR-4 - if enhancing "capsule" \\ - LR-5 - if nonperipheral "washout" OR threshold growth}

Figure 1. LI-RADS Diagnostic Table. Reproduced with permission from the American College of Radiology LI-RADS version 2018 manual. Available from: https//www.acr.org/Clinical-resources/Reporting-and-Data-Systems/LI-RADS ${ }^{[4]}$. LI-RADS: Liver Imaging Reporting and Data System

for the evaluation of contrast-enhanced ultrasound, screening ultrasound, and CT and MRI following locoregional therapy for HCC. LI-RADS v2018 also includes technical recommendations for the performance of CT and MRI for the evaluation of HCC. The development of LI-RADS has been in the context of patients with cirrhosis, chronic hepatitis B infection without cirrhosis, or a history of prior HCC. LI-RADS should not be used in patients with vascular causes for their cirrhosis due to the decreased specificity of arterial hyperenhancement for malignancy in these patients ${ }^{[3]}$.

\section{LI-RADS V2018}

The LI-RADS algorithm provides a step by step approach for consistent categorization of observations seen on CT and MR imaging of patients with cirrhosis, chronic hepatitis B viral infection, or a history of $\mathrm{HCC}^{[4]}$. Observations that are definitely benign (LR-1) or probably benign (LR-2) can be categorized based on typical imaging features associated with specific benign entities, such as hepatic hemangiomas, cysts, or perfusion alterations. Observations that demonstrate imaging features suggestive of malignancies other than HCC are categorized as LR-M. These imaging features include peripheral washout appearance, targetoid restricted diffusion, and rim arterial phase hyperenhancement. Observations that are not initially categorized as LR-NC, LR-1, LR-2, LR-M, or LI-RADS Tumor in Vein (LR-TIV) are then categorized using the LI-RADS Diagnostic Table [Figure 1]. The diagnostic table guides the radiologist to category LR-3, LR-4, or LR- 5 based on the presence or absence of major features as discussed in further detail below. 
Table 2. LI-RADS v2018 minimum technical recommendations for CT

\begin{tabular}{ll}
\hline Feature & \multicolumn{1}{c}{ Recommendation } \\
\hline Scanner configuration & $\geq 8$ detector rows \\
Multiplanar reformations & Suggested \\
Slice thickness & $\leq 5 \mathrm{~mm}$ required for axial reconstructions \\
& $3-2.5 \mathrm{~mm}$ suggested for multiplanar reformations if obtained \\
Precontrast imaging & Suggested for patients that have had prior locoregional therapy, optional otherwise \\
Contrast-enhanced phases & Late arterial \\
& Portal venous \\
& Delayed $(2-5 \mathrm{~min})$ \\
Contrast administration & Injection rate of $\geq 3 \mathrm{~mL} / \mathrm{s}$ \\
& $\geq 300 \mathrm{mgl} / \mathrm{mL}$ for dose of $1.5-2.5 \mathrm{~mL} / \mathrm{kg}$ \\
& Saline chaser bolus $(30-40 \mathrm{~mL})$ \\
\hline
\end{tabular}

Adapted with permission from American College of Radiology Liver Imaging Reporting and Data System version 2018 manual. Available from: https//www.acr.org/Clinical-resources/Reporting-and-Data-Systems/LI-RADS ${ }^{[4]}$. LI-RADS: Liver Imaging Reporting and Data System; CT: computed tomography

Although an update to LI-RADS was released in 2017, a new version was released only a year later to allow for two changes to CT/MRI LI-RADS. Both changes were made to allow for unification between LI-RADS and American Association for the Study of Liver Disease (AASLD) practice guidelines. The first change was the definition of threshold growth. Previously, threshold growth was defined as $\geq 50 \%$ size increase of a mass in $\leq 6$ months, $\geq 100 \%$ increase in size over $>6$ months, or a new $\geq 10 \mathrm{~mm}$ observation developing in $\leq 24$ months. The definition of threshold growth in LI-RADS v2018 is now restricted to only $\geq 50 \%$ increase in size of a mass over $\leq 6$ months. This stricter definition is now concordant with growth criteria used by the AASLD and the Organ Procurement and Transplantation Network (OPTN). The second change was to the categorization of 10-19 mm observations with arterial phase hyperenhancement and one additional major feature in the LI-RADS Diagnostic Table. Previously, these observations were categorized based on which imaging features were present and required the use of "-g" and "-us" added to the LR-5 to specify the features used. This portion of the table has now been simplified so that if a 10-19 mm observation with arterial phase hyperenhancement also demonstrates nonperipheral "washout" or threshold growth, it is categorized as LR-5. If a 10-19 mm observation with arterial phase hyperenhancement only demonstrates the additional feature of an enhancing "capsule"; however, it is designated as LR-4.

\section{TECHNICAL RECOMMENDATIONS}

Consistent imaging techniques are necessary to enable reproducibility of LI-RADS categories between radiologists at different institutions. The wide variety of equipment, technical parameters, and sequences available throughout the world can lead to difficulty in evaluating critical imaging features for LI-RADS category assessment. To address these inconsistencies, the Technique Working Group of LI-RADS has developed minimum technical requirements for the performance of CT and MRI in patients at risk for developing HCC, summarized in Tables 2 and $3^{[5]}$.

\section{Vascular phases for CT and MRI}

Multiphase contrast enhanced imaging is required to make the imaging diagnosis of HCC (LI-RADS 5) confidently and adequately evaluate the regional vascular anatomy and patency. Pre-contrast imaging provides information on pre-existing hyperattenuating and T1 hyperintense material, which are often seen as a sequelae of locoregional therapies. This bright material can obscure or mimic enhancement on postcontrast phases. Although pre-contrast T1-weighted imaging is required for MRI, a pre-contrast phase is optional for CT imaging unless the patient has had locoregional therapy due to the low likelihood of pre-existing hepatic high attenuation material in a patient without locoregional therapy and associated increased radiation from an additional CT imaging phase. 
Table 3. LI-RADS v2018 minimum technical recommendations for MRI

\begin{tabular}{ll}
\hline Feature & \multicolumn{1}{c}{ Recommendation } \\
\hline Scanner strength & 1.5 or 3 Tesla \\
Coil type & Phased array multichannel torso coil \\
Slice thickness & $\leq 5 \mathrm{~mm}$ required for multiphase contrast-enhanced sequences \\
& $\leq 8 \mathrm{~mm}$ with $\leq 2 \mathrm{~mm}$ slice gap for other sequences \\
Multiphase contrast-enhanced & T1 weighted with 3D acquisition and fat suppression strongly recommended \\
phases (required) & Precontrast \\
& Late arterial \\
& Portal venous \\
& Delayed (2-5 min) if using ECA or gadobenate \\
Contrast administration & Transitional phase (2-5 min) if using gadoxetate \\
& Hepatobiliary phase (approx 20 min) if using gadoxetate \\
& ECA or gadobenate or gadoxetate, weight adjusted dose \\
& Injection rate of 1-2 mL/s \\
& Saline chaser bolus (30-40 mL) \\
Other required sequences & Unenhanced T1-weighted opposed-phase and in-phase imaging \\
& T2-weighted imaging (fat suppression optional) \\
Other suggested sequences & Diffusion weighted imaging \\
& Multiplanar acquisitions, such as coronal T2-weighted imaging \\
\hline
\end{tabular}

Adapted with permission from American College of Radiology Liver Imaging Reporting and Data System version 2018 manual. Available from: https//www.acr.org/Clinical-resources/Reporting-and-Data-Systems/LI-RADS ${ }^{[4]}$. LI-RADS: Liver Imaging Reporting and Data System; MRI: magnetic resonance imaging; ECA: extracellular contrast agent

The first required post-contrast phase is the late hepatic arterial phase which refers to the phase of contrast when there is enhancement of the portal vein but no antegrade enhancement of the hepatic veins. As some HCCs are not conspicuous until the late hepatic arterial phase, earlier arterial phase imaging can result in reduced sensitivity for $\mathrm{HCC}^{[6]}$. The arterial phase is also required for evaluation of the LI-RADS major feature of arterial phase hyperenhancement.

The portal venous phase occurs when antegrade enhancement of the portal and hepatic veins is present and there is peak parenchymal enhancement of the liver. Portal venous phase imaging is often the phase that best demonstrates the LI-RADS major feature of "washout appearance" due to the peak enhancement of background liver.

Delayed phase refers to the imaging phase performed after the portal venous phase, typically 3-5 min following the injection of an extracellular contrast agent (ECA) or gadobenate. The vessels and parenchyma all remain enhanced during this phase, but are overall decreased in brightness compared to the portal venous phase. A combination of the portal venous phase and delayed phase can more reliably demonstrate the LI-RADS major features of "washout appearance" and "capsule appearance" than the portal venous phase alone ${ }^{[7]}$. Examples of these dynamic imaging phases are shown in Figure 2.

The transitional and hepatobiliary phases are only available on MRI following the administration of hepatobiliary contrast agents (HBAs). Hepatobiliary contrast agents such as gadobenate and gadoxetate are gadolinium contrast agents that have a portion of the contrast agent enter the hepatocytes with subsequent excretion into the biliary system. The transitional phase occurs between the portal venous phase and hepatobiliary phase and demonstrates persistent vascular enhancement with increasing hepatic parenchymal enhancement. Although both gadobenate and gadoxetate are capable of demonstrating a hepatobiliary phase, gadobenate does not have sufficiently strong parenchymal update to allow for a transitional phase. The hepatobiliary phase refers to a delayed phase following HBA administration that shows hyperintensity of hepatic parenchyma and relative hypointensity of the hepatic vessels. This phase provides high sensitivity for observations that do not have hepatocytes capable of contrast uptake, which includes a broad spectrum of both benign and malignant entities ${ }^{[7]}$. Patients with severe hepatic dysfunction, however, may not have sufficient hepatocyte uptake of the contrast agent to yield an adequate 

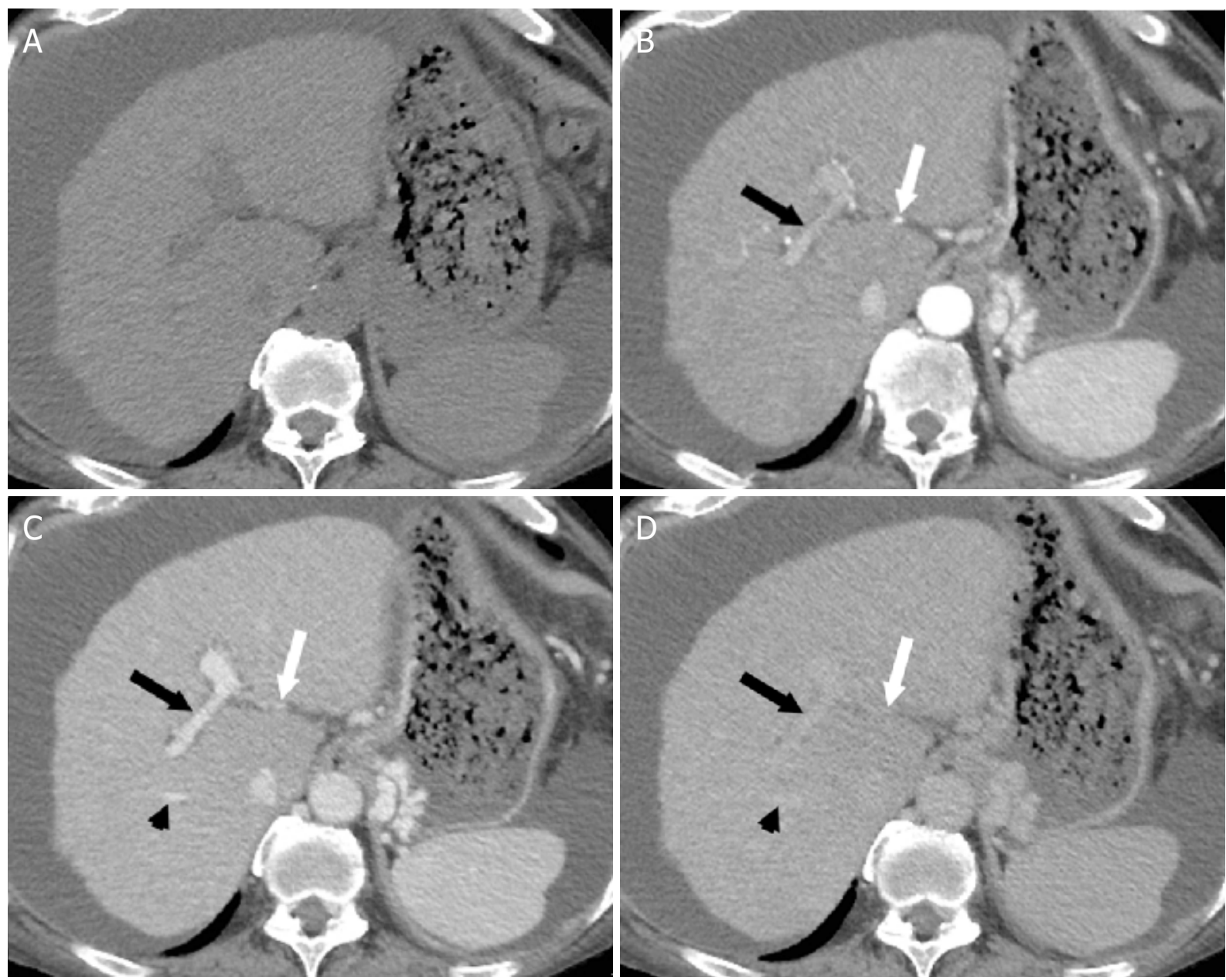

Figure 2. Dynamic Imaging for Evaluation for Hepatocellular Carcinoma. Axial CT images demonstrating the pre-contrast (A), hepatic arterial (B), portal venous (C), and delayed (D) post-contrast imaging phases. Pre-contrast imaging (A) is not required for $\mathrm{HCC}$ evaluation unless the patient has undergone local ablation that results in high attenuation material in the liver that may obscure enhancing lesions. The hepatic artery (white arrow) and portal vein (black arrow) demonstrate enhancement during the hepatic arterial phase (B). During the portal venous phase (C), the hepatic veins begin to enhance (black arrowhead). The hepatic veins, portal veins, and hepatic arteries demonstrate progressively decreasing enhancement during the delayed phase (D). HCC: hepatocellular carcinoma; CT: computed tomography

hepatobiliary phase, and a history of prior inadequate hepatobiliary phase imaging should be factored in the selection of contrast agents for future imaging in these patients.

\section{Additional MRI sequences}

In addition to dynamic contrast enhanced imaging, T2-weighted and unenhanced T1-weighted opposedphase and in-phase imaging are required for complete evaluation of the liver in patients at risk for HCC. These sequences provide important information regarding the presence of fat, iron, fibrosis, and edema in observations relative to the background liver parenchyma that can contribute to observation conspicuity and enable evaluation for the presence of ancillary features that can enable modification of the final LI-RADS category.

Diffusion weighted imaging provides information regarding relative cellularity of tissues and is also an important sequence for improving the specificity and sensitivity for $\mathrm{HCC}^{[8]}$. Despite the widespread recognition of the importance of diffusion weighted imaging in this setting, however, this sequence remains optional since it is not as widely available and robust as other MRI sequences ${ }^{[5]}$. An example of an MR exam that meets complete technical requirements for LI-RADS is shown in Figure 3. 

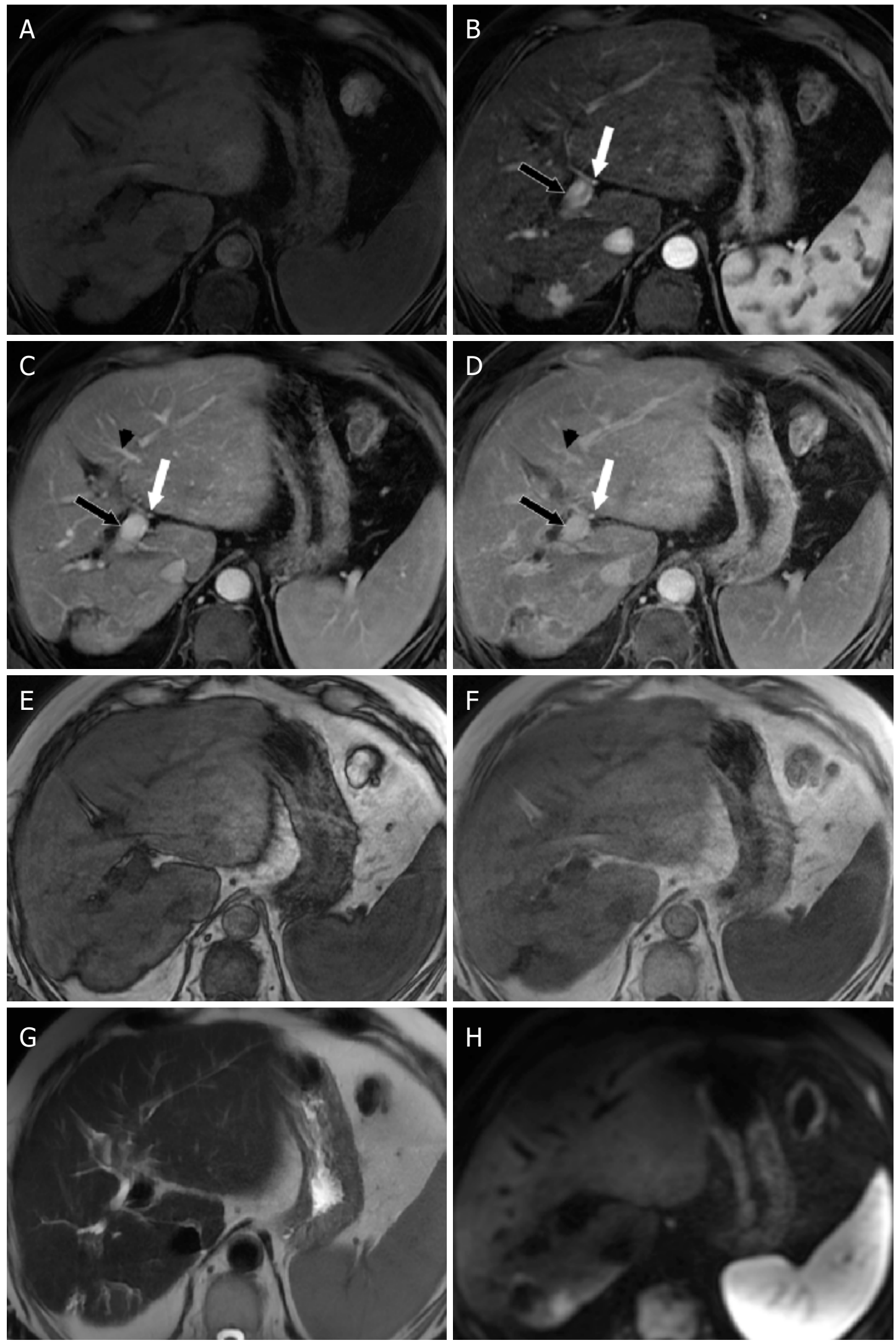

$\mathrm{H}$

Figure 3. MRI for Evaluation for HCC. Axial MR images of an exam meeting minimum technical requirements for evaluation of HCC. Axial T1-weighted fat saturated images should be obtained pre-contrast ( $A$ ), and following intravenous contrast during the hepatic arterial (B), portal venous (C), and delayed (D) phases. Additional required imaging sequences include opposed phase (E) and in phase (F) T1-weighted images and T2-weighted images (G). Diffusion weighted imaging $(H)$ is not required, but is suggested if available. White arrow: hepatic artery; Black arrow: portal vein; Black arrowhead: hepatic vein. MRI: magnetic resonance imaging; HCC: hepatocellular carcinoma 


\section{IMAGING FEATURES}

\section{Major features}

LI-RADS major features are the primary imaging features used to categorize observations as LR-3, LR-4, or LR-5. Major features were selected to provide high specificity for hepatocellular carcinoma and are the only features that can be used to categorize an observation as LR-5. These major features are included in the diagnostic table and include arterial phase hyperenhancement, nonperipheral "washout", enhancing "capsule", size, and threshold growth [Figure 1].

Arterial phase hyperenhancement refers to enhancement of an observation during the arterial phase that is greater than the background liver and results in signal intensity or attenuation that is higher than the background liver. This feature is best assessed during the late hepatic arterial phase and is present in most HCCs that have progressed ${ }^{[9,10]}$. A peripheral pattern to the arterial phase hyperenhancement, however, has been associated with non-HCC malignancies such as cholangiocarcinoma and metastases [Figure 4] ${ }^{[11]}$. Therefore, only nonrim arterial phase hyperenhancement should be used for the assignment of LR-5 category to an observation.

The term nonperipheral "washout" refers to reduction in enhancement in whole, or in part, within an observation from an earlier post-contrast imaging phase to a later extracellular post-contrast phase. "Washout" (with quotation marks) refers to visual assessment of washout appearance and does not specifically require measurement of enhancement or construction of an enhancement curve. "Washout" in combination with arterial phase hyperenhancement is a highly specific imaging feature of HCC ${ }^{[12,13]}$. If "washout" is present primarily along the margins of the observation, however, the imaging feature is instead considered peripheral "washout" and is not a major feature due to its association with intrahepatic cholangiocarcinoma ${ }^{[11]}$. Hypointensity on the transitional or hepatobiliary phases should not be considered "washout" since the high specificity of washout in the literature and its inclusion in LI-RADS has been based on exams performed with ECA. If "washout" is present in an observation prior to the transitional phase on an exam using a hepatobiliary contrast agent, "washout" can be considered present and used as a major feature for LI-RADS categorization.

Enhancing "capsule" describes a smooth uniform border around the majority of an observation margin that is unequivocally thicker or distinct from any fibrotic tissue present elsewhere in the liver. To be considered a major feature, this finding must be present on the portal venous, delayed, or transitional phase of postcontrast imaging [Figure 5]. The term "capsule" is used in place of capsule appearance, since the imaging finding of a "capsule" can be indicative of either a true fibrous capsule or pseudocapsule on histology. Regardless of whether a true capsule or pseudocapsule is present, however, the imaging feature of "capsule" is present in $12 \%-94 \%$ of $\mathrm{HCCs}^{[14-17]}$

The size of an observation is the largest outer edge to outer edge dimension of an observation and should be measured on the sequence or phase where the margins of the observation are the most clear and distinct. Due to perfusion alterations that can manifest during the arterial phase, size should not be assessed on the arterial phase unless the observation is not visible on any other phase or sequence. Also, due to anatomic distortion that is often present on diffusion weighted imaging (DWI), measurements should be avoided on the DWI sequence unless the observation is not visible on another sequence. If the observation demonstrates capsule appearance, the capsule should be included in the size measurement. Only observations that are $10 \mathrm{~mm}$ or larger are eligible to be considered definitely as HCC (LR-5) in combination with arterial phase hyperenhancement and other major features.

Threshold growth in LI-RADS v2018 refers to the size increase of an observation by greater than $50 \%$ within six months. Threshold growth only applies to observations that are definitely masses, since perfusion 

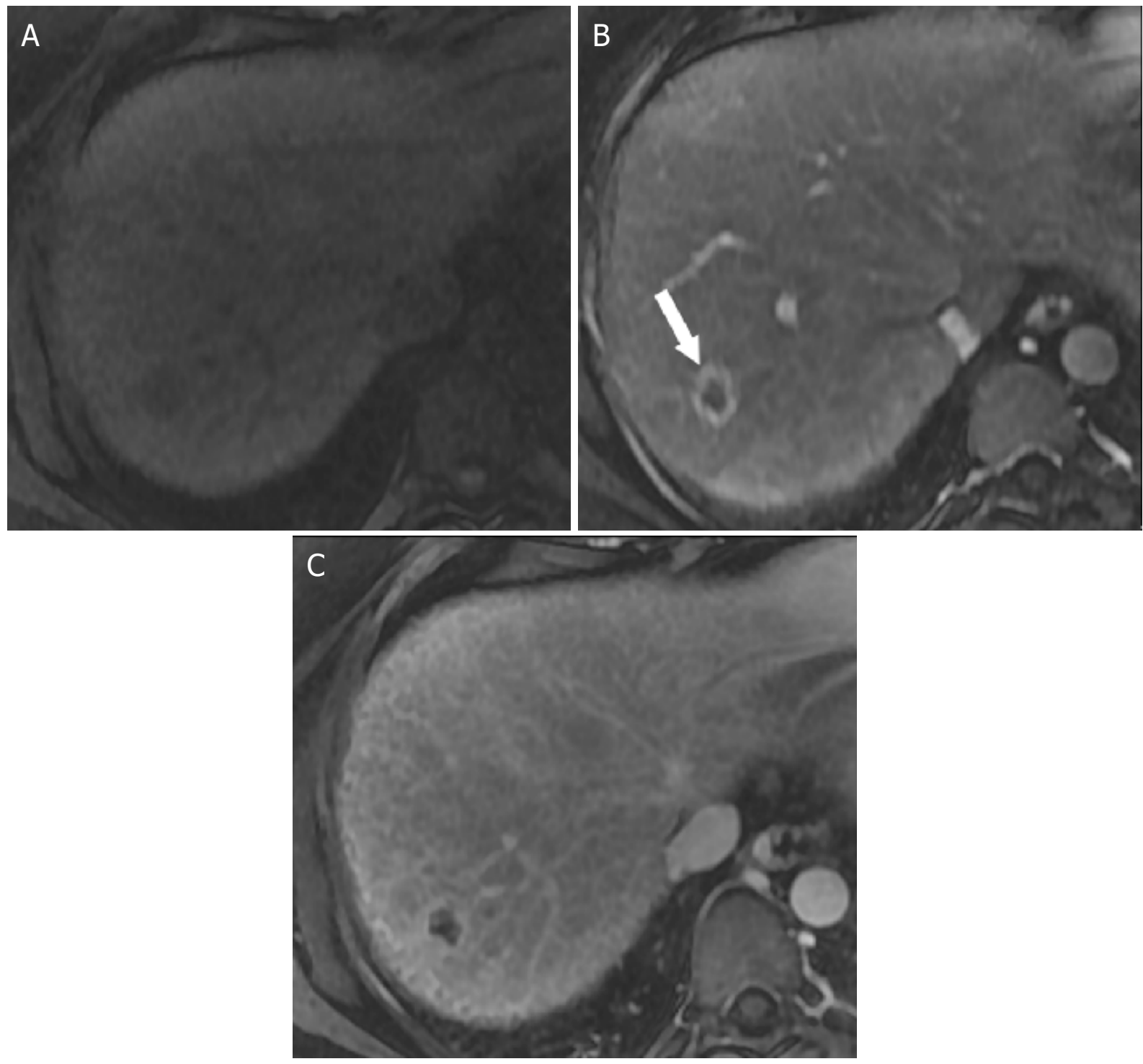

Figure 4. Peripheral Arterial Phase Hyperenhancement. Axial T1-weighted fat saturated MR images of the abdomen prior to (A), and during the arterial phase (B) and delayed phase (C) of dynamic post extracellular contrast images. The $26 \mathrm{~mm}$ observation in the right hepatic lobe demonstrates peripheral arterial phase hyperenhancement (white arrow). This feature does not qualify as the major feature of nonrim arterial phase hyperenhancement and should lead the radiologist to assign the category of LR-M (probably or definitely malignancy, but not HCC specific). HCC: hepatocellular carcinoma

alterations can often vary in size from one exam to the next. Also, the comparison prior examination must be a CT or MRI exam that was performed 6 months or less prior to the more recent study [Figure 6]. This definition of threshold growth is different from prior versions of LI-RADS and was changed to achieve congruence with the definition of threshold growth used by the OPTN ${ }^{[18]}$. Note that the development of a new observation within 6 months of a prior examination is not considered threshold growth in LI-RADS, as the definition requires that the observation was present on the prior exam.

\section{Ancillary features}

Ancillary features are those imaging features that can be used to change the LI-RADS category of an observation after the application of major features. Ancillary features can change the category by one category to reflect either a higher or lower suspicion of malignancy. Ancillary features cannot, however, be used to change the category of an observation from LR-4 to LR-5. Only major features may be used 

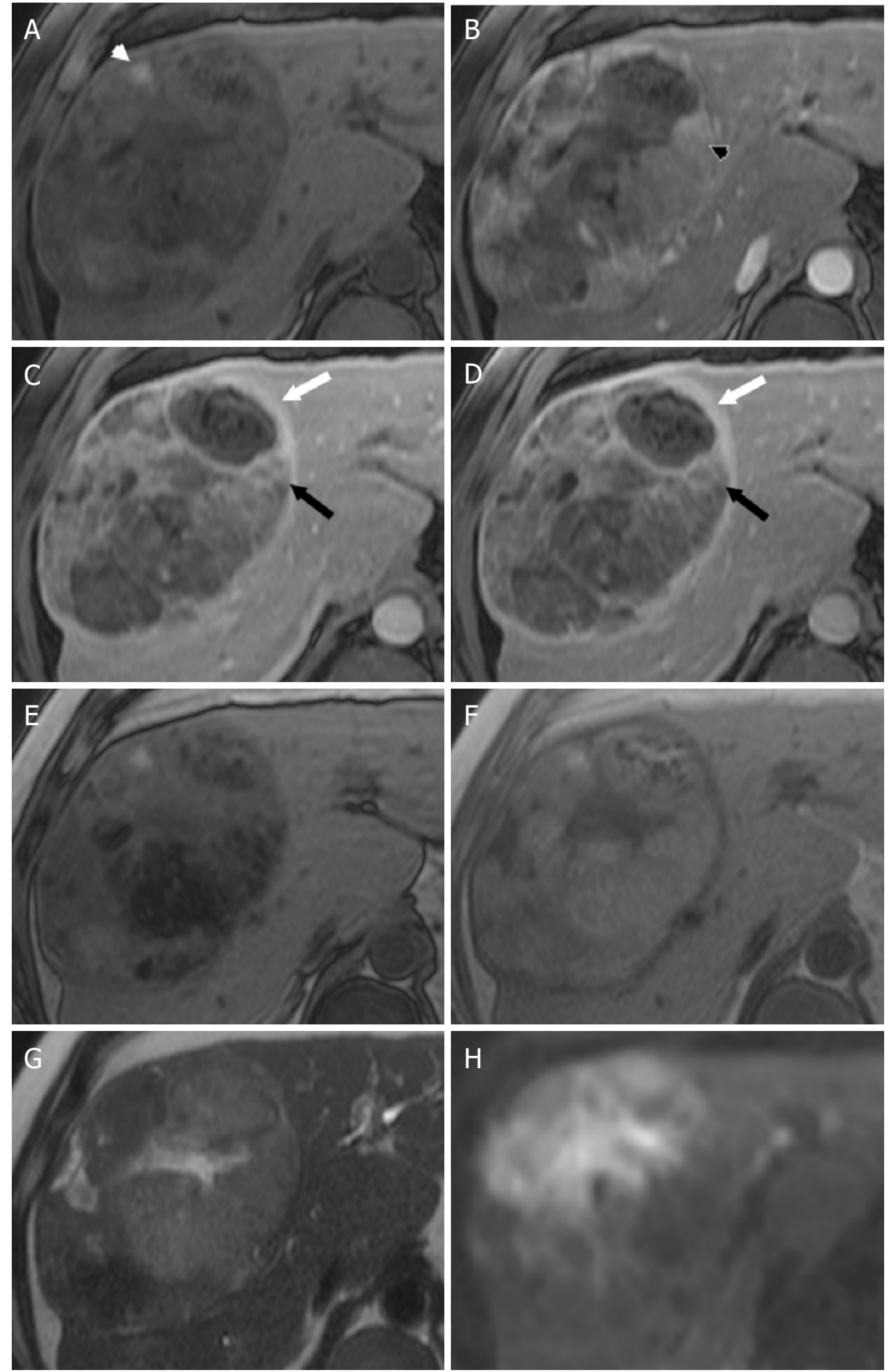

Figure 5. LR-5 - Definitely HCC. Axial MR images of a $14 \mathrm{~cm}$ mass at the junction of the right and left hepatic lobes. T1-weighted fat saturated images performed prior to (A) as well as during the arterial phase (B), portal venous phase (C), and delayed phase (D) following contrast administration demonstrate areas of arterial phase hyperenhancement (black arrowhead), "washout" (black arrow), and "capsule" (white arrow). The presence of three major features and size greater than $20 \mathrm{~mm}$ lead to LI-RADS 5 as the correct category for this mass. Additionally, the mass has ancillary features favoring HCC including mosaic architecture, fat in mass (seen as loss of signal between in-phase (F) and opposed-phase (E) T1-weighted images), and blood products in mass (white arrowhead, A). Additional ancillary features favoring malignancy, not HCC in particular, are also present including mild-moderate T2 hyperintensity (T2-weighted image, G) and restricted diffusion (diffusion weighted images, H). HCC: hepatocellular carcinoma 

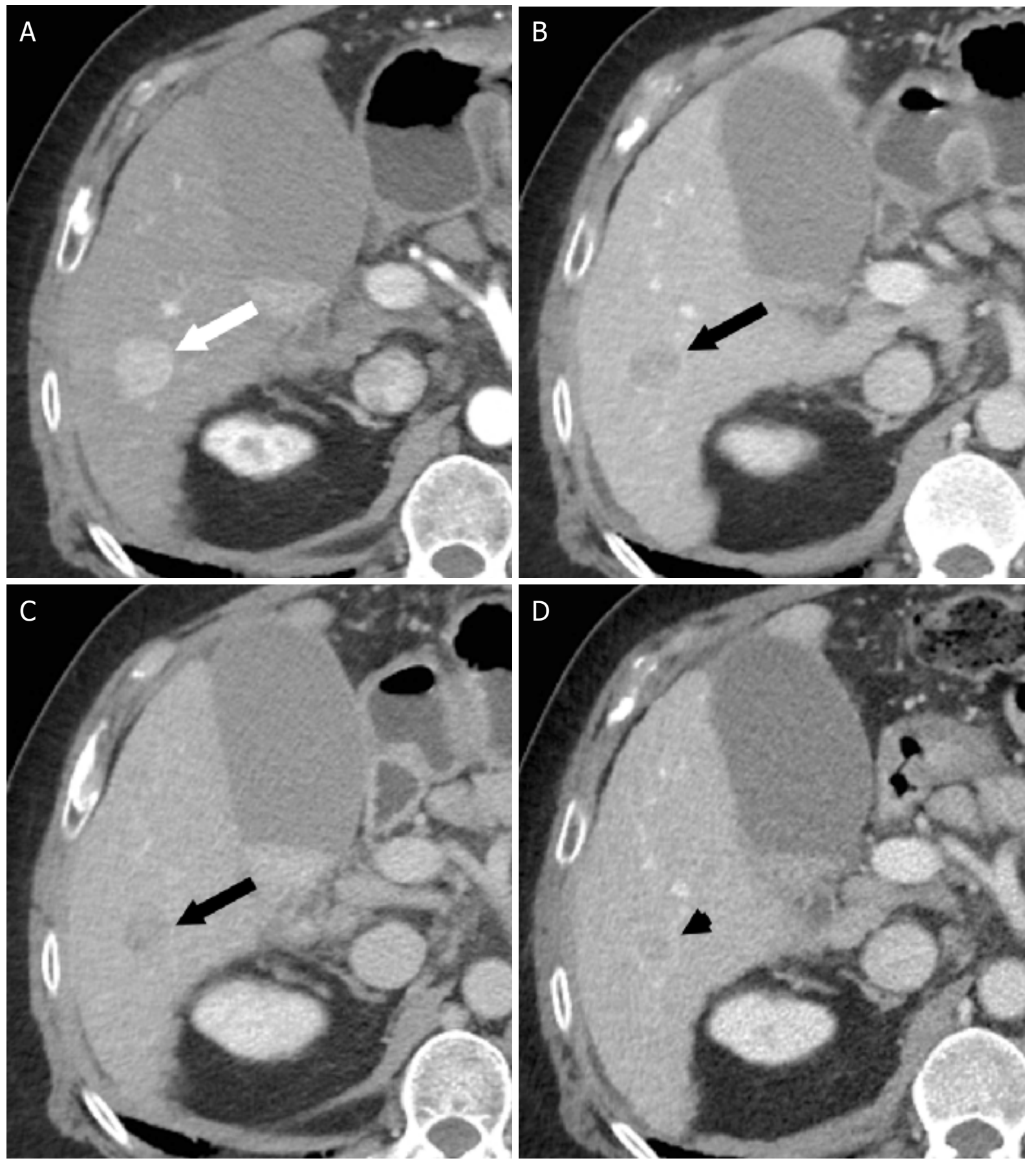

Figure 6. Threshold Growth. Axial CT images of a $21 \mathrm{~mm}$ mass in the right hepatic lobe. The mass demonstrates arterial phase hyperenhancement during the arterial phase (white arrow, A), and "washout" with "capsule" (black arrow) on the portal venous (B) and delayed phases (C). Axial CT performed 5 months prior demonstrates a $12 \mathrm{~mm}$ mass on the portal venous phase (D, black arrowhead). The interval growth is more than $50 \%$ in less than 6 months, therefore, the mass also demonstrates threshold growth. The most appropriate category for this mass is LI-RADS 5. CT: computed tomography; LI-RADS: Liver Imaging Reporting and Data System

to categorize an observation as LR-5 to preserve high specificity for HCC. In LI-RADS v2018, ancillary features are divided into those suggestive of malignancy versus those suggestive of benignity [Table 4]. Ancillary features suggestive of malignancy are further subdivided into those that are and are not specific to HCC. For example, the presence of intralesional fat is considered a specific finding of HCC in those patients at risk for developing HCC, whereas restricted diffusion can be present in many types of malignant 


\section{Table 4. LI-RADS v2018 ancillary imaging features}

\begin{tabular}{lll}
\hline Features favoring malignancy, not HCC in particular & Features favoring HCC in particular & Features favoring benignity \\
\hline US visibility as discrete nodule & Nonenhancing "capsule" & Size stability $\geq 2$ years \\
Subthreshold growth & Nodule-in-nodule architecture & Size reduction \\
Corona enhancement & Mosaic architecture & Parallels blood pool enhancement \\
Fat sparing in solid mass & Fat in mass, more than adjacent liver & Undistorted vessels \\
Restricted diffusion & Blood products in mass & Iron in mass, more than liver \\
Mild-moderate T2 hyperintensity & & Marked T2 hyperintensity \\
Iron sparing in solid mass & & Hepatobiliary phase isointensity \\
Transitional phase hypointensity & \\
Hepatobiliary phase hypointensity & \\
\hline
\end{tabular}

RADS: Liver Imaging Reporting and Data System; HCC: hepatocellular carcinoma
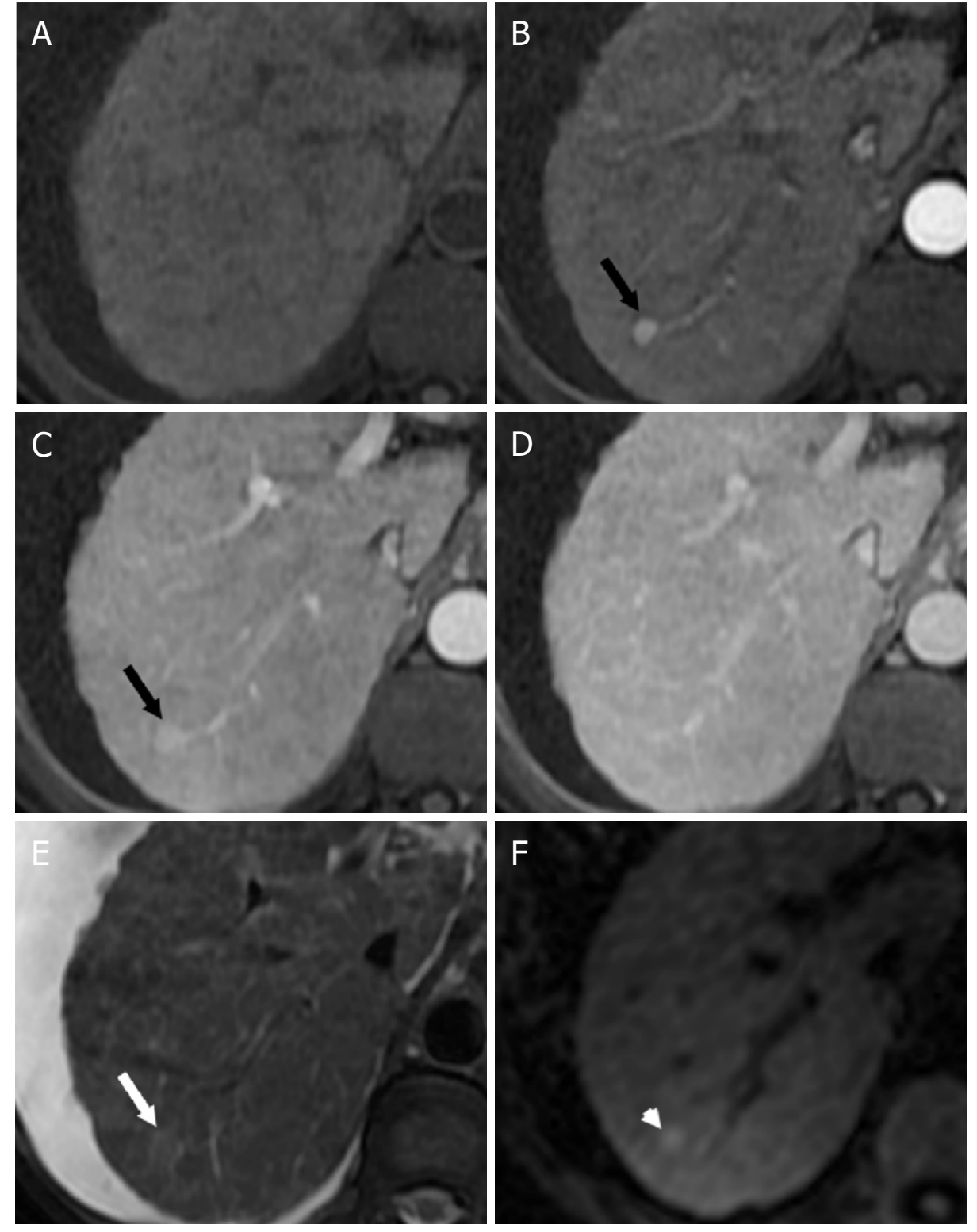

Figure 7. LR-4 - Probably HCC. Axial MR images of a $9 \mathrm{~mm}$ observation in the right hepatic lobe. T1-weighted fat saturated images performed prior to (A) as well as during the arterial phase (B), portal venous phase (C), and delayed phase (D) following contrast administration demonstrate arterial phase hyperenhancement (black arrow) of the observation which persists into the portal venous phase. Based on the size and presence of a single major feature, the appropriate category for this observation is LI-RADS 3. The observation also demonstrates mild T2-hyperintensity (white arrow, E) and restricted diffusion (white arrowhead, F). The presence of ancillary features suggestive of malignancy then allow the radiologist discretion with changing the category to LI-RADS 4 to reflect higher suspicion for HCC. HCC: hepatocellular carcinoma; LI-RADS: Liver Imaging Reporting and Data System 

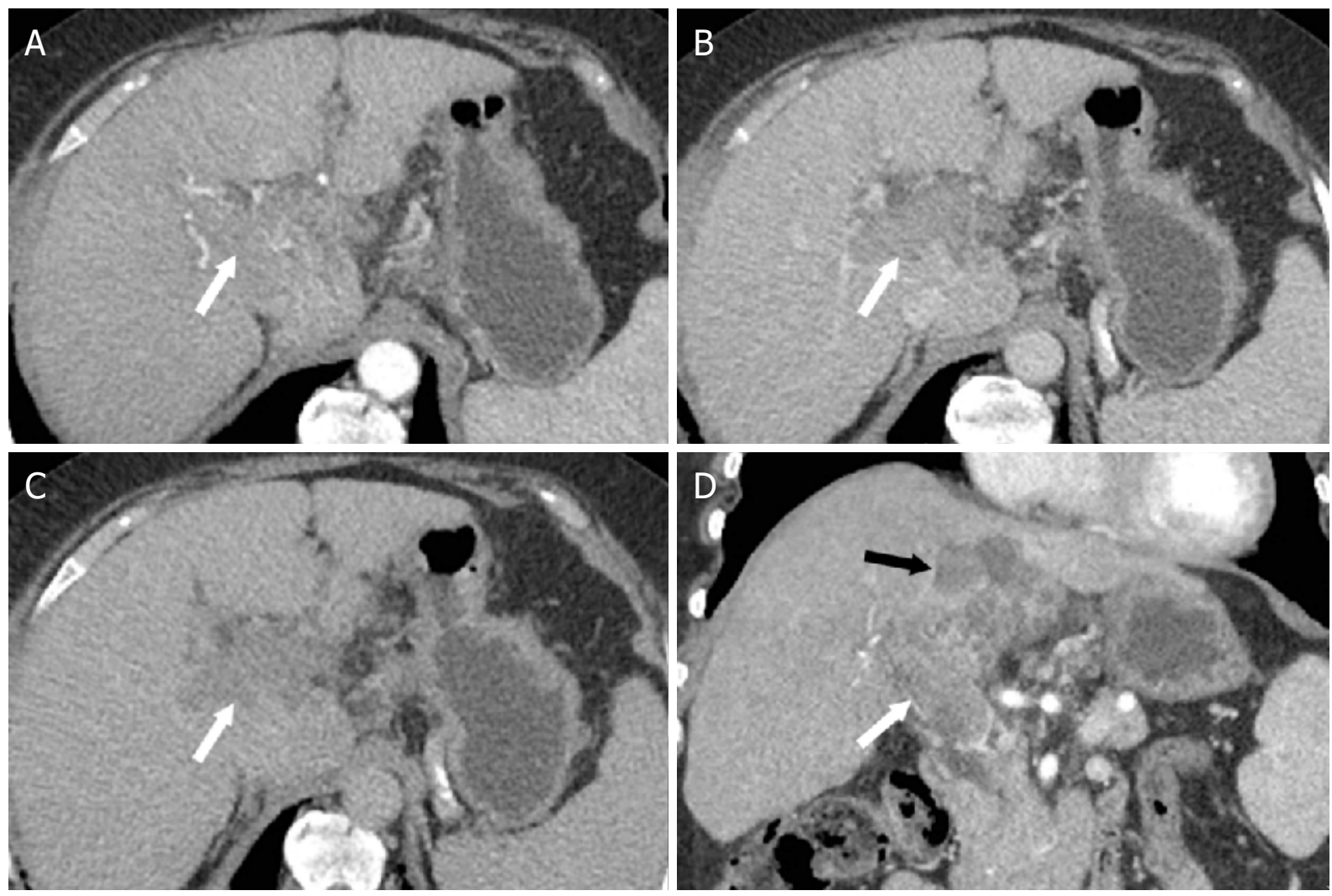

Figure 8. LR-TIV. Axial CT images from the hepatic arterial (A), portal venous (B), and delayed (C) phases following intravenous contrast administration and coronal image from the hepatic arterial phase (D). Enhancing soft tissue is present in the right and main portal veins (white arrow). Due to an associated parenchymal mass (black arrow) with arterial phase hyperenhancement (not shown) and "washout", the most appropriate category for this finding is LR-TIV, likely due to HCC. CT: computed tomography; LI-RADS: Liver Imaging Reporting and Data System; HCC: hepatocellular carcinoma; LR-TIV: LI-RADS Tumor in Vein

lesions such as metastases from an extrahepatic primary malignancy [Figure 7$]^{[8]}$. If an observation has multiple ancillary features for both benignity and malignancy, then the category of the observation should not be adjusted. Finally, the use of ancillary features is optional at the radiologist's discretion for designating a LI-RADS category.

\section{TUMOR IN VEIN}

The category of LR-TIV should be used for the evidence of vascular invasion of the portal veins or hepatic veins. Unequivocal vascular invasion is present when enhancing soft tissue is present within the vessel [Figure 8]. This category does not require the presence of a parenchymal mass. If possible, the radiologist should indicate the most probably etiology between HCC and non-HCC malignancy.

\section{CONCLUSION}

Since its initial release in 2011, CT/MR LI-RADS has evolved based on user feedback, ongoing expert review, and the need for unification with other HCC imaging algorithms. CT/MR LI-RADS v2018 provides an algorithm for the standardized reporting and interpretation of findings in patients at risk for HCC, and is now concordant with AASLD practice guidelines. With increasing adoption worldwide, the CT/MR algorithm and associated lexicon enable clearer communication between radiologists, other physicians, and researchers to better provide care for patients at risk for developing HCC. 


\section{DECLARATIONS}

\section{Authors' contributions}

The author contributed solely to the article.

\section{Availability of data and materials}

Not applicable.

\section{Financial support and sponsorship}

None.

\section{Conflicts of interest}

The author declared that there are no conflicts of interest.

\section{Ethical approval and consent to participate}

Not applicable.

\section{Consent for publication}

Not applicable.

\section{Copyright}

(C) The Author(s) 2020.

\section{REFERENCE}

1. Elsayes KM, Kielar AZ, Chernyak V, Morshid A, Furlan A, et al. LI-RADS: a conceptual and historical review from its beginning to its recent integration into AASLD clinical practice guidance. J Hepatocell Carcinoma 2019;6:49-69.

2. Sirlin CB. The LI-RADS adventure-a personal statement. Abdom Radiol (NY) 2018;43:1-2.

3. Chernyak V, Santillan CS, Papadatos D, Sirlin CB. LI-RADS® algorithm: CT and MRI. Abdom Radiol (NY) 2018;43:111-26.

4. American College of Radiology. Liver Imaging Reporting and Data System version 2018. Available from: http://www.acr.org/QualitySafety/Resources/LIRADS. [Last accessed on 12 Aug 2020]

5. Kambadakone AR, Fung A, Gupta RT, Hope TA, Fowler KJ, et al. LI-RADS technical requirements for CT, MRI, and contrast-enhanced ultrasound. Abdom Radiol (NY) 2018;43:56-74.

6. Ichikawa T, Kitamura T, Nakajima H, Sou H, Tsukamoto T, et al. Hypervascular hepatocellular carcinoma: can double arterial phase imaging with multidetector CT improve tumor depiction in the cirrhotic liver? AJR Am J Roentgenol 2002;179:751-8.

7. Choi JY, Lee JM, Sirlin CB. CT and MR imaging diagnosis and staging of hepatocellular carcinoma: part II. Extracellular agents, hepatobiliary agents, and ancillary imaging features. Radiology 2014;273:30-50.

8. Chernyak V, Tang A, Flusberg M, Papadatos D, Bijan B, et al. LI-RADS ${ }^{\circledR}$ ancillary features on CT and MRI. Abdom Radiol (NY) 2018;43:82-100.

9. Matsui O, Kobayashi S, Sanada J, Kouda W, Ryu Y, et al. Hepatocelluar nodules in liver cirrhosis: hemodynamic evaluation (angiography-assisted CT) with special reference to multi-step hepatocarcinogenesis. Abdom Imaging 2011;36:264-72.

10. Muto J, Shirabe K, Sugimachi K, Maehara Y. Review of angiogenesis in hepatocellular carcinoma. Hepatol Res 2015;45:1-9.

11. Fowler KJ, Potretzke TA, Hope TA, Costa EA, Wilson SR. LI-RADS M (LR-M): definite or probable malignancy, not specific for hepatocellular carcinoma. Abdom Radiol (NY) 2018;43:149-57.

12. Pawlik TM, Delman KA, Vauthey JN, Nagorney DM, Ng IO, et al. Tumor size predicts vascular invasion and histologic grade: implications for selection of surgical treatment for hepatocellular carcinoma. Liver Transpl 2005;11:1086-92.

13. Forner A, Vilana R, Ayuso C, Bianchi L, Solé M, et al. Diagnosis of hepatic nodules 20 mm or smaller in cirrhosis: prospective validation of the noninvasive diagnostic criteria for hepatocellular carcinoma. Hepatology 2008;47:97-104.

14. Kelekis NL, Semelka RC, Worawattanakul S, de Lange EE, Ascher SM, et al. Hepatocellular carcinoma in North America: a multiinstitutional study of appearance on T1-weighted, T2-weighted, and serial gadolinium-enhanced gradient-echo images. AJR Am J Roentgenol 1998;170:1005-13.

15. Freeny PC, Baron RL, Teefey SA. Hepatocellular carcinoma: reduced frequency of typical findings with dynamic contrast-enhanced CT in a non-Asian population. Radiology 1992;182:143-8.

16. Kadoya M, Matsui O, Takashima T, Nonomura A. Hepatocellular carcinoma: correlation of MR imaging and histopathologic findings. Radiology 1992;183:819-25.

17. Choi JY, Lee JM, Sirlin CB. CT and MR imaging diagnosis and staging of hepatocellular carcinoma: part I. Development, growth, and 
spread: key pathologic and imaging aspects. Radiology 2014;272:635-54.

18. Kielar AZ, Chernyak V, Bashir MR, Do RK, Fowler KJ, et al. An update for LI-RADS: Version 2018. Why so soon after version 2017? J Magn Reson Imaging 2019;50:1990-1. 